P0120

\title{
LIGHT SCIENCE IS NOT ONLY SCIENCE OF LIGHTING: THEORETICAL BASES AND APPLICATION AREA

\author{
Julian Aizenberg et al.
}

DOI 10.25039/x46.2019.PO120

from

CIE x046:2019

Proceedings

of the

29th CIE SESSION

Washington D.C., USA, June 14 - 22, 2019

(DOI 10.25039/x46.2019)

The paper has been presented at the 29th CIE Session, Washington D.C., USA, June 14-22, 2019. It has not been peer-reviewed by CIE.

(c) CIE 2019

All rights reserved. Unless otherwise specified, no part of this publication may be reproduced or utilized in any form or by any means, electronic or mechanical, including photocopying and microfilm, without permission in writing from CIE Central Bureau at the address below. Any mention of organizations or products does not imply endorsement by the CIE.

This paper is made available open access for individual use. However, in all other cases all rights are reserved unless explicit permission is sought from and given by the $\mathrm{CIE}$.

CIE Central Bureau

Babenbergerstrasse 9

A-1010 Vienna

Austria

Tel.: +4317143187

e-mail: ciecb@cie.co.at

www.cie.co.at 


\title{
LIGHT SCIENCE IS NOT ONLY SCIENCE OF LIGHTING: THEORETICAL BASES AND APPLICATION AREA
}

\author{
Aizenberg J.B. ${ }^{1}$, Budak V.P. ${ }^{2}$ \\ ${ }^{1}$ Lighting Research Institute (VNISI), Moscow, RUSSIA, \\ ${ }^{2}$ National research university "MPEI", Moscow, RUSSIA \\ budakvp@mpei.ru
}

DOI 10.25039/x46.2019.PO120

\begin{abstract}
At present, the areas of application of light for illumination and in non-visual, technological purposes has become almost equal. These areas of application have a unified theoretical basis - a ray-based light field theory. Today the connection of all theories of light: quantum, wave, and ray - has been fully disclosed. Each theory is valid in its field of application and does not need any clarification. A single theoretical basis of all light applications requires a new definition of the lighting engineering specialty, which, in turn, will require its organizational design. The necessity of a corresponding change in the structure of the CIE is shown.
\end{abstract}

Keywords: Light Field, Ray, Optical Receiver, Light Technology, Wave Content of Light Field, Non-visual Light Effects

\section{Introduction}

The science of light is currently facing two fundamental and long overdue challenges. First, lighting engineering has long ceased to be only the science of electric lighting. The development of light sources and methods of spatial control of luminous fluxes are continually expanding the field of application of lighting engineering systems. In addition to the visual and non-visual effects of light on humans, the following intensively developing areas of the optical radiation use should be noted:

- technological use in agriculture;

- physiotherapy effects on the human body and the widest use of light in medicine;

- use of the UV part of the optical spectrum in irradiating installations for purifying drinking water, disinfecting room volumes, and deodorizing the air;

- problems of solar energy with the conversion of natural light into electric current with its subsequent accumulation and conversion into light;

- optical remote sensing of Earth surface and cloud layer;

- information transmission using light radiation;

- lighting design;

and many other applications.

Such widespread use of lighting systems defines a second challenge: the formulation of the theoretical bases of this science. All the diverse areas of light use are united by a common theoretical basis - the unified physical principles of the optical radiation propagation. It constitutes the essence of the light field in the framework of ray photometric concepts about light.

This paper is devoted to the study of the theoretical foundations and the identification of the application fields of modern lighting engineering. 


\section{Theory of light field}

The introduction of electric lighting into our lives necessitated the development of methods for calculating lighting systems, methods for measuring their characteristics, and determining the norms of lighting to perform an activity. A theoretical model of light was created for this. The theory of (Bouguer, 1729) - (Lambert, 1760) was based on Kepler's ideas (Kepler, 1604) about light as a combination of rays, so this model was called ray or photometric. The model was completed by (Gershun, 1936) in the light field theory: he studies the space from the standpoint of transmission of radiant energy within that space.

The development of the theory of the light field coincided with the development of the wave theory of light, and later with the development of quantum electrodynamics. The connection between the theory of a light field, the wave and quantum nature of light remained unclear for a long time and thus was regarded in physics as purely approximate, engineering, applied.

The central concept of the light field theory - the ray seemed entirely alien for electrodynamics. In the history of the development of physics, the ray and wave natures of light have always been opposed to each other. The triumph of Maxwell's electromagnetic theory led to the rejection of radiation representations to the roadside of physics. The emergence of quantum electrodynamics in some sense breathed life into the ray representations, but this gave rise to the so-called wave-particle duality. A real understanding of the connection of all theories of light has opened an understanding of the role of radiation measurements by an optical receiver (Rozenberg, 1977).

In field theory, we study the specific that distinguishes a given point in space from others and thereby turns space into a field. To study the field at each point in space is introduced a test receiver. According to his reaction, each point of space is given a specific value, which forms the field. However, in the case of light, there are two types of receivers: linear, the response of which is a force, and quadratic, which reacts to the power of the field at this point, which is quadratic concerning the force. Thus, it is possible to construct two theories of the light field: an electromagnetic field theory or otherwise wave optics based on a linear receiver, and based on a quadratic power receiver, which is commonly called the theory of a light field.

Note that since energy (power) is a derivative of a force, all states of the light field can be derived from wave optics, and in this sense wave optics is a more general theory. Wave optics operates with quantities not observed in the experiment - the amplitude and phase of the wave field. The light field is formulated exclusively in terms of the observed values - irradiation and brightness. Pure wave phenomena such as diffraction and interference in the experiment manifest themselves in a specific distribution of irradiance. Therefore, any description of the experiment in the framework of wave optics necessarily leads to the need to go to the light field.

In the works of the late twentieth century, the connection of the light field with the wave theory of light and quantum electrodynamics was revealed and resulted in a hierarchy of three approaches to the nature of light. The highest of them is the quantum theory which explains all currently known light phenomena and is based on the representation of light as an ensemble of photons. This theory is the most abstract and therefore the most difficult to use to interpret real measurements. If the number of photons in the ensemble is huge, the movement can be described as a wave process, which naturally leads to wave optics.

In principle, the wave and ray descriptions are equivalent to each other in the region of wave homogeneity: at each point the ray is perpendicular to the wave. Therefore, knowing the wavefront in space, one can always restore the rays and vice versa. The condition of homogeneity (quasi-uniformity) of the field is a small change in the field on a wavelength scale (Apresyan, 1996). However, the field is non-uniform and the ray description is impossible if the wavefront changes dramatically at the wavelength scale (e.g. the diffraction of light on a small hole).

The revealed connection of the three light theories showed that within their limits each model forms a closed approach and does not need any clarification. Moreover, the photometric, ray model is correct in most practical examples, and differences from the model are manifested in very subtle experiments. 


\section{New determination of light engineering}

The light field theory is significantly beyond the scope of lighting technology and serves as a language for describing almost any technological use of light. Therefore, the scale of the lighting science is incomparably more significant than only the questions of lighting. And where are the boundaries of research in the field of lighting engineering, and how do they conditionally separate lighting engineering from optics, laser technology, radio engineering, and astronomy, for example?

The lighting science covers the transfer of only incoherent radiation based on the laws of the light field. And this is the primary and fundamental characteristic of any lighting research, development and their use.

The purpose and content of lighting engineering is to develop the science of light in the framework of radiation, photometric concepts and to apply the results for comfort lighting, as well as for technological purposes, e.g. medicine. Light engineering is a field of science and technology and its subject is development of methods for generation and spatial redistribution of optical radiation, as well as its conversion to other forms of energy and use for various purposes.

Currently, the area of non-visual light effects (different technological field of light application) is actively and widely developed. At the same time, this significant area of light application is outside the zone of attention of the scientific headquarters of lighting engineering - CIE. Light engineering must answer the main challenges of our time: there is not enough water, food, and energy.

One of the most effective ways of providing the population of the world with drinking water is disinfection of natural water within the framework of irradiation installation with UV radiation. Such powerful installations have been created and used in many countries of the world, providing water to the population of multi-million cities.

Cultivation of agricultural products is impossible without greenhouses, where artificial lighting plays a crucial role in growing the crop, which also serves for heating. Irradiation plants of greenhouses are distinguished by a wide light distribution, a spectral distribution that is specific for each crop, and which changes with the time of plant growth.

The most important role in the development of humanity is the solution to the energy problem. One of the essential areas for solving the energy problem is renewable energy sources, among which solar energy plays a unique role. The efficiency of such sources largely depends on the light redistribution systems of sunlight. The problem of bringing light inside the room, creating installations of combined illumination, also abuts here.

Along with this, today the non-visual effects of light also include the use of light technologies in medicine, welding and cutting of materials, information transmission, and the development of the ocean and space.

All these technologies are united by a single theoretical base, a single conceptual point of view - the theory of the light field. For the development of the theory of the light field and its applications, it is desirable to guide this process from a single center. The development of lighting engineering in various applied areas leads to a duplication of research, and sometimes to logically contradictory results.

\section{Conclusions}

$\mathrm{XXI}$ century is the century of light. The scope of light is continuously expanding. The presence of a single international scientific and technical approach will allow considering the fundamental theoretical and applied problems in all areas of the use of light from a unified scientific position. Essentially the CIE should include further development of the lighting engineering science into its activities plan, primarily to ensure a unified approach of specialists from different countries in our science and the formation of training programs in the field of lighting engineering. 
Active development of the areas of non-visual light effects requires the study and synthesis of accumulated experience on radiation sources, optical systems, and special materials. CIE does not deal with these sections since it was created as an organization summarizing the experience in the field of illumination, and the area of applications of non-visual exposure to light may soon become an equal area of illumination. Therefore, it is desirable that the newly created groups work closely with existing specialized international organizations, creating with them joint technical committees.

Along with this, the most important program of $\mathrm{CIE}$ development is to attract a wide range of specialists from different countries. Existing scientific and technical journals can play an important role here. To coordinate the activities of which the coordinating body of the CIE is needed.

\section{References}

APRESYAN, L.A., KRAVTSOV, YU.A. 1996. Radiation Transfer: Statistical and Wave Aspects. Netherlands: Gordon and Breach Publishers.

BOUGUER, P. 1729. Pierre Bouguer's Optical Treatise on The Gradation of Light / translated, with introduction and notes by W.E. Knowles Middleton. Toronto: University of Toronto Press, 1961.

GERSHUN A., 1936. The Light Field. Moscow. Trans. P. Moon and G. Timoshenko. Journal of Mathematics and Physics, 18, MIT, 1939, 51-151.

KEPLER, J. 1604. Ad Vitellionem paralipomena. Frankfurt: C. Marnium \& Hæredes Aubrii.

LAMBERT, J.H. 1760. Photometria sive de mensura et gradibus luminis, colorum et umbrae / Lamberts Photometrie. Ostwald's Klassiker der Exakten Wissenschaften, No. 31, 32, 33. Leipzig: Engelmann, 1892.

ROZENBERG, G.V. 1977. The light ray (contribution to the theory of the light field). Sov. Phys. Usp., 20, 55-80. 